\title{
An Evolutionary Interpretation of Teleostean Forebrain Anatomy
}

\author{
Thomas Mueller $^{\mathrm{a}}$ Mario F. Wullimann ${ }^{\mathrm{b}}$ \\ a University of California San Francisco, Department of Biopharmaceutical Sciences, School of Pharmacy, \\ San Francisco, Calif., USA; ${ }^{\text {b }}$ Ludwig-Maximilians-University, Department Biology II - Neurobiology, \\ Graduate School of Systemic Neurosciences, Laboratory of Prof. Dr. B. Grothe, Planegg-Martinsried, \\ Munich, Germany
}

\section{Key Words}

Amygdaloid complex - Basal ganglia - Isocortex • LGE • MGE $\cdot$ Migration $\cdot$ Pallidum $\cdot$ Striatum

\begin{abstract}
During the past few years, our investigations of the forebrain in the zebrafish (a teleost fish) have shown that its molecular anatomy and expression patterns of genes involved in the regulation of neuronal transmitter phenotypes, such as $\gamma$ aminobutyric acid- (GABA-)ergic neurons, are very similar to those seen in mammalian model organisms such as mouse and rat. For example, we have been able to identify previously undiscovered homologies, such as subpallial regions in the zebrafish that are homologous to the medial and lateral ganglionic eminences in mammals, as well as regions homologous to the larval eminentia thalami and its adult derivative, the bed nucleus of the stria medullaris. Furthermore, in what we term the partial eversion model of the telencephalon in teleosts, we propose homologies to all four mammalian pallial areas and conclude that the posterior zone of the dorsal telencephalic area in teleosts is homologous to the piriform cortex and is formed by a migratory stream of cells originating in a dorsomedial zone of the pallium (the primordial medial zone of area dorsalis telencephali). In this review we critically discuss and justify these findings in the context of forebrain evolution in fishes.
\end{abstract}

\section{KARGER}

Fax +4161306 1234

E-Mail karger@karger.ch

www.karger.com (c) 2009 S. Karger AG, Basel

0006-8977/09/0741-0030\$26.00/0

Accessible online at:

www.karger.com/bbe

\section{Introduction}

In this paper we attempt to compare teleostean and mammalian forebrains. Such an endeavor could easily be disastrous, as is amply documented in the literature. For example, a direct topographical comparison of telencephalic cell masses in adult teleosts and adult mammals clearly leads to erroneous conclusions because teleost telencephala are everted, rather than evaginated like mammalian telencephala, resulting in a significant topographical rearrangement of structures [see Nieuwenhuys, 2009]. We believe that one can avoid such sins of interpretation, however, by using an approach founded on the views of Karl Ernst von Baer who uttered the 'single most important words in the history of comparative embryology' [Gould, 1977]:

(1) The general features of a large group of animals appear earlier in the embryo than the special features.

(2) Less general characters are developed from the most general, and so forth, until finally the most specialized appear.

(3) Each embryo of a given species, instead of passing through the stages of other animals, departs more and more from them.

(4) Fundamentally therefore, the embryo of a higher animal is never like the adult of a lower animal, but only like its embryo [von Baer, 1828; as translated by Gould, 1977]. 


\begin{tabular}{ll}
\hline Abbreviations used in this paper \\
\hline ac & anterior commissure \\
CGE & caudal ganglionic eminence \\
cp & cerebral peduncle \\
Dc & central zone of area dorsalis telencephali \\
DC & central posterior thalamic nucleus \\
Dl & lateral zone of area dorsalis telencephali \\
Dm & medial zone of area dorsalis telencephali \\
Dp & posterior zone of area dorsalis telencephali \\
DP & dorsal posterior thalamic nucleus \\
DT & dorsal thalamus \\
E & epiphysis \\
EmT & eminentia thalami \\
H & hypothalamus \\
Ha & habenula \\
lfb & lateral forebrain bundle \\
LGE & lateral ganglionic eminence \\
LP & lateral pallium \\
LV & lateral telencephalic ventricle \\
MGE & medial ganglionic eminence \\
MP & medial pallium \\
MV & midbrain ventricle \\
P & pallium (larval) \\
Po & preoptic region \\
Pr & pretectum \\
PSB & pallial-subpallial boundary \\
PTd & dorsal part of posterior tuberculum \\
PTv & ventral part of posterior tuberculum \\
Sd & dorsal division of subpallium (larval) \\
Sdd & dorsal territory of Sd (striatum) \\
Sdv & ventral territory of Sd (pallidum) \\
Sv & ventral division of subpallium (larval) \\
T & tegmentum \\
TeO & tectum opticum \\
TV & telencephalic ventricle \\
3V & third ventricle \\
Vd & dorsal nucleus of area ventralis telencephali \\
VT & ventral thalamus \\
VP & ventral pallium \\
Vv & ventral nucleus of area ventralis telencephali \\
ZLI & zona limitans intrathalamica \\
\hline
\end{tabular}

Therefore, in the early development of the forebrain in vertebrates as phylogenetically remote as a zebrafish and a mouse, we can expect to find the corresponding structures based on the presence of a common Bauplan for vertebrate brains. It is well documented that this Bauplan reflects many similarities in development among vertebrates, and it has recently been expanded as the neuromeric model [Puelles and Rubenstein, 1993, 2003]. This model can be viewed as a three-dimensional grid of histogenetic units [Puelles and Medina, 2002] whose topo- logical arrangement is highly conserved, evolutionarily, compared to its anteroposterior, dorsoventral and mediolateral molecular coordinates. Here, histogenetic units are defined as radial territories that give rise to molecularly coherent cell groups that share a developmental origin, a developmental history, and an adult fate. This approach leads to possibly the clearest definition of neural homology we have encountered: In any two vertebrate species, brain structures are homologous if: (1) their development can be traced back to the same topological origin within the periventricularly located proliferative matrix; (2) they share a similar developmental history, defined by similar stages and the expression of regulatory and other genes; and (3) they are defined in the adult by a similar set of neuronal (transmitter/neuromodulator) phenotypes as a result of the preceding developmental events. Any proposed homology should also be tested with outgroup comparisons, however, to ensure the uninterrupted phylogenetic descent of the structure in question [see Northcutt, 1984, for an introduction of the cladistic method to neurobiology].

We strongly support the idea that the recognition of a homology is independent of function, such as connectivity. Thus, information in addition to developmental and molecular genetic data - for example, data on physiological function or neural connectivity - may provide support for proposed homologies (homologous structures can be similar in function), but it cannot determine homologies alone or refute them. For example, a functional difference, such as a light-sensitive epiphysis in various vertebrates with axonal projections to brain structures versus a solely endocrine epiphysis in primates, or pronounced differences in pallial connections of the dorsal thalamus in amniotes and teleosts, cannot be cause for rejecting a proposed homology that is based on developmental and molecular data and is plausibly integrated into the Bauplan and a cladistic scenario. We argue that these latter characteristics form a logical hierarchy for defining a neural structure and recognizing its homologue in other species. This hierarchy would extend from place and origin of a structure in the Bauplan to the molecular genetic pathway associated with it and the resulting neurochemical characteristics, followed by more derivative phenotypic features, such as connections and nuclear morphology, which are more prone to change during phylogeny. Additional confirmation of phylogenetic continuity using outgroup comparison is of course necessary for recognizing a homology. If, hypothetically, only mammals and sharks had a cerebellum, we would conclude that it originated convergently 
twice independently, although both would fulfill the developmental criteria mentioned for recognizing a homology.

In order to identify histogenetic units in the telencephalon and characterize their full radial extent, we initially looked at early proliferation and neurogenesis with molecular markers. That is, we looked at the expression patterns of genes and the distribution of proteins that define certain stages of neuronal cells in the entire developing brain (not just the forebrain) [Ma et al., 1997; Casarosa et al., 1999; Fode et al., 2000; Bertrand et al., 2002]. By relating the expression patterns of proneural genes involved in the determination and differentiation of neuronal cells, to the zones of proliferation and neuronal differentiation, we intended to follow the radial fate of a neuronal phenotype from its site of origin (proliferative matrix) up to its final location in the radial periphery of the grey matter of a given histogenetic unit. One notable deviation from this radial concept of the development of histogenetic units is the phenomenon of tangential migration, such as that of the nascent $\gamma$-aminobutyric acid- (GABA-)ergic interneurons that originate in the subpallium and migrate tangentially into the cortex of mammals [Marín and Rubenstein, 2001; Nery et al., 2002] or the rostral migratory stream from the subpallial-pallial ventricular boundary zone into the olfactory bulb [Hack et al., 2005; Lledo et al., 2006]. As we will see below, both radial and tangential migratory processes are reflected by gene expression patterns in the developing zebrafish brain, and these patterns can be compared with those in other vertebrate species, including mammals.

The patterns observed in the zebrafish are also consistent with known differences in early brain development in teleosts and mammals: for example, the processes of telencephalic eversion in teleosts and telencephalic evagination in mammals, and the resulting different locations of subpallial and pallial telencephalic divisions in the two taxa. In addition, these differences can be used to explain the migrating streams related to telencephalic divisions in teleosts, streams whose courses were altered, topographically, by eversion, but still maintaining the same topological relationships. It is highly implausible that these similar developmental patterns arose twice, independently, or that they would differ in the outgroup of teleosts and mammals: the cartilaginous fishes. In fact, studies on embryonic telencephalic cell migrations in cartilaginous fishes [Rodríguez-Moldes, 2009] are in general agreement with our findings.

\section{Evolutionarily Conserved Patterns of Forebrain Neurogenesis}

In order to identify molecular anatomical homologies in the developing zebrafish (teleostean) forebrain and to define developmental stages that correspond to forebrains of mammalian model organisms such as mouse and rat, we initially studied zebrafish expression patterns of those regulatory and other genes known to be involved in the determination and differentiation of neuronal cells. These molecular markers included Notch and Delta orthologues and genes coding for basic helix-loop-helix (bHLH) transcription factors [Blader et al., 1997; Dornseifer et al., 1997; Appel and Eisen, 1998; Korzh et al., 1998; Bertrand et al., 2002; Ross et al., 2003]. The initial results showing the anatomical distribution of these markers during the early phase of secondary neurogenesis in the forebrains of early developing zebrafish larvae, i.e., two to five days postfertilization, were compared to available mouse data for the period of mammalian primary neurogenesis (roughly mouse E9.0 to E18.5) published by various labs [Ma et al., 1997; Horton et al., 1999; Schuurmans and Guillemot, 2002]. Primary neurogenesis in very early zebrafish (before $24 \mathrm{~h}$ ) has already been described and is mostly that of transitorily generated neurons, such as the Rohon-Beard cells. Therefore, herein we will solely address secondary postembryonic zebrafish neurogenesis [see Mueller and Wullimann, 2003, 2005; Wullimann and Mueller, 2004a]. The diverse expression patterns of zebrafish and mice were compiled and mapped onto schematic representations of their early forebrains, and the comparison suggested the existence of a phylotypic stage of neurogenesis during the early development of vertebrate brains [Wullimann and Mueller, 2004a, Mueller et al., 2006], as corroborated by new data from mice [Osório et al., 2009]. The results on which these conclusions are based will be discussed below in chronological order.

\section{Molecular Characterization of Early Proliferation and Proneural Gene Expression Patterns}

First indications that there are patterns of secondary neurogenesis in teleostean forebrains that are similar to those of primary neurogenesis in mammalian forebrains came from our studies of expression patterns of neurogenin1 (ngn1) and NeuroD in comparison to sites of proliferation visualized either by immunohistochemistry for the proliferating cell nuclear antigen (PCNA) or for previ- 
Fig. 1. Schematic drawings of rostral (upper left) to caudal (lower right) transverse sections through the forebrain of a twoday-old zebrafish showing bHLH gene expression. Compiled data show Zashla (black; sites of GABA origin) and ngn1/ neuroD (gray) gene expression domains where supposedly predominantly glutamatergic neurons are produced. Dlx $2 a$ expression is in Zashla regions, except for ventral posterior tuberculum and pretectum. * = Pax6 positive ventral part of ventral thalamus, which likely contains ventrally migrating GABA/GAD- and THexpressing cells. See list for abbreviations. [After Wullimann and Mueller, 2002; Mueller and Wullimann, 2003].

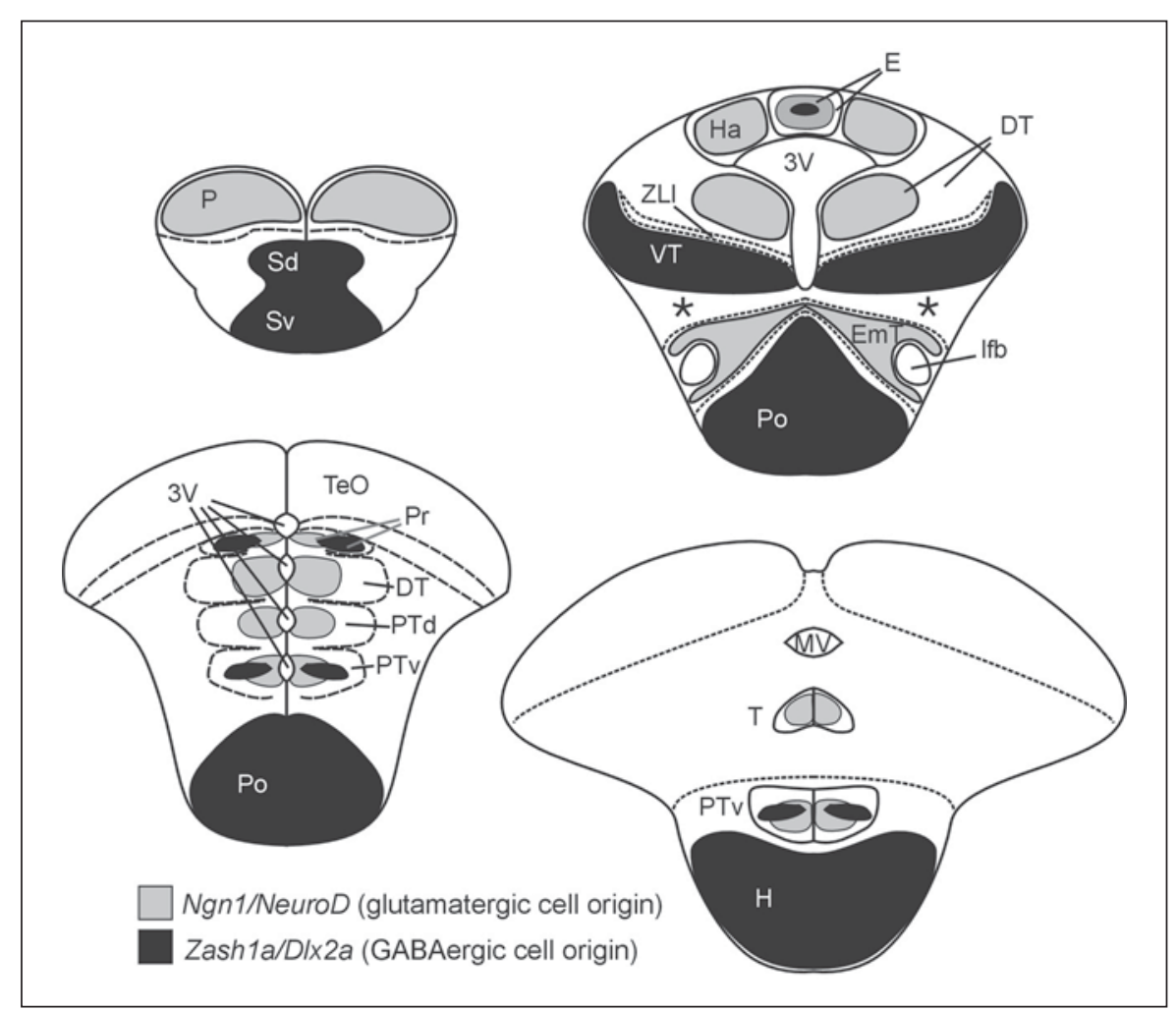

ously introduced bromodeoxyuridine (BrdU) in the developing zebrafish forebrain at two to five days [Mueller and Wullimann, 2002, 2003]. During this time frame, ngnl and NeuroD are likely involved in the massive production of glutamatergic neurons (see below). These genes are expressed in slightly overlapping patterns that appear adjacent to the proliferative matrix of many - but not all - forebrain regions. Specifically, expression sites of $n g n 1$ and NeuroD include the olfactory bulb (OB), the pallium (P), the pretectum (Pr), the dorsal thalamus (DT), the eminentia thalami (EmT), the dorsal posterior tuberculum (PTd), and the ventral posterior tuberculum (PTv), which reaches caudoventrally into the dorsal part of the inferior lobe. Four other forebrain regions were defined by the absence of $n g n 1$ and NeuroD: the subpallium (S), the preoptic region (Po), the ventral thalamus/prethalamus (VT), and the hypothalamus (H) [Mueller and Wullimann, 2002, 2003, 2005; Wullimann and Mueller, 2002]. In general, the expression domains of $n g n 1$ and NeuroD confirmed the composition of the early postembryonic zebrafish (i.e., teleost) brain - three prosomeres (P1-P3), pretectum, dorsal thalamus, ventral thalamus (fig. 1) previously revealed by proliferation studies [Wullimann and Puelles, 1999] - plus an eminentia thalami (EmT), which is molecularly different from the (ngn1/NeuroD negative) ventral thalamus, as well as different from the remaining, complexly organized, most anterior, secondary prosencephalon. In contrast, the original prosomeric model suggested that six prosomeres comprise the entire forebrain [Puelles and Rubenstein, 1993], and it was later modified to include three prosomeres (P1-3) plus a secondary prosencephalon, with the eminentia thalami forming a part of P3 [Puelles and Rubenstein, 2003].

The forebrain regions that were defined by the absence of $n g n 1$ and NeuroD expression (S, Po, VT, H) showed expression of Zashla instead. The Zashla gene is the zebrafish orthologue of the Mash1 gene in mammals, which is involved in the specification of GABAergic cells (fig. 1) [Allende and Weinberg, 1994; Wullimann and Mueller, 2002]. Within vertebrate forebrains, $D l \times 2$ is another gene involved in the specification of GABAergic cells [Anderson et al., 1997; Panganiban and Rubenstein, 2002; Flames et al., 2007].

The Dlx2 is a homeobox gene (distal less), which is restricted to subpallial, preoptic, ventral thalamic and hypothalamic regions of the forebrain in mice [Marín et al., 2000; Flames et al., 2007; Zhao et al., 2008], and the orthologous gene in zebrafish, $D l \times 2 a$, is expressed in all 
Fig. 2. Schematic drawings of transverse sections through the telencephalon of an early zebrafish (left) and a mouse at a comparable stage (right), indicating the molecular organization of teleostean basal ganglia and the topographically medial tangential migratory stream of GABAergic cells invading the pallium, compared to the condition in mammals, with a topographically lateral migratory stream. Some adult pallial divisions in the zebrafish are indicated for a better understanding of the text. The course of pallial radial glia fibers is indicated (on the left side) in both the zebrafish and the mouse. See list for abbreviations. [Left panel after Wullimann and Rink, 2002; Mueller et al., 2006, 2008. Right panel after Grigoriou et al., 1998; Puelles et al., 2000; Flames et al., 2007].

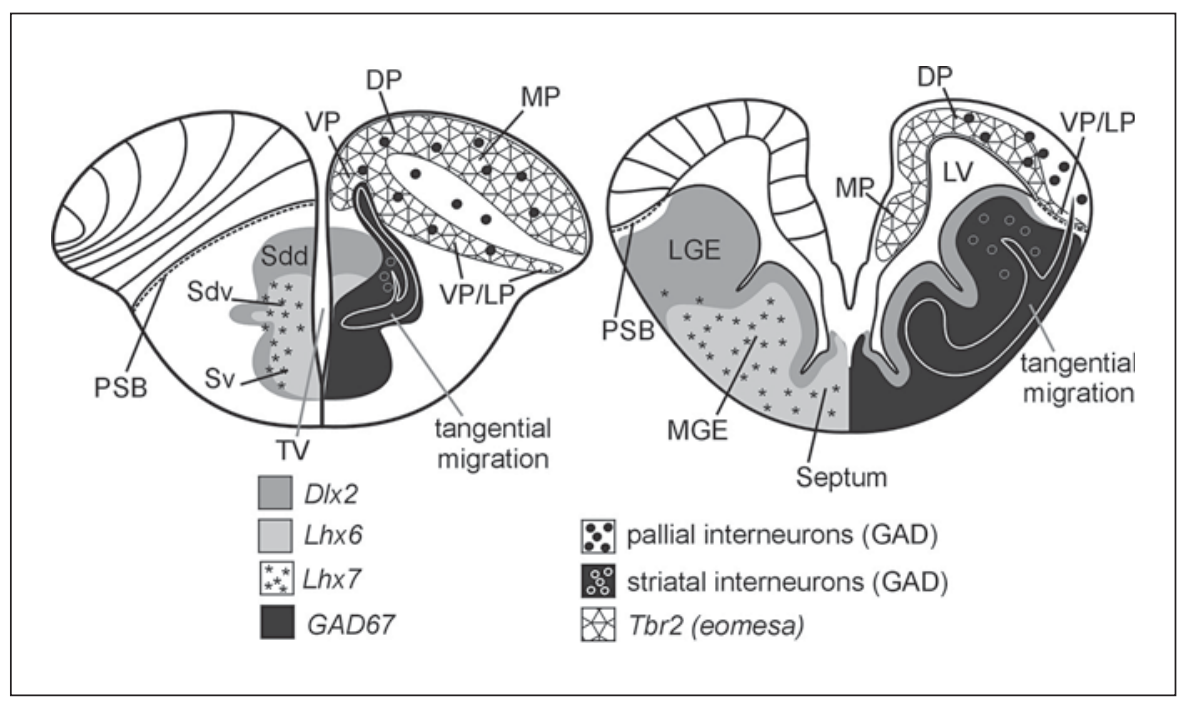

four homologous regions (fig. 1) that define the major sites of forebrain GABAergic cell production [Mueller et al., 2008]. Thus, the overall picture emerging from these gene expression studies is that $D l \times 2 a$ and Zash1a, which are involved in the massive production of GABAergic cells, are expressed in a pattern that is complementary to that of genes involved in the production of glutamatergic cell types in the zebrafish forebrain, but there are certain regions that probably express Zash $1, a$ as well as $n g n 1$ and NeuroD in a different manner, and we call this phenomenon the expression switched pattern. Regions with the expression switched pattern are: the pretectal region (Pr; alar plate prosomere 1); and the ventral posterior tuberculum (PTv; basal plate prosomere 3). Overall, the complementary expression of Zashla, neurogenin, and NeuroD in the forebrains of two- to three-day-old zebrafish embryos highly resembles the expression patterns of the orthologous genes (Mash1, neurogenin1, NeuroD) in mouse brains between embryonic stages E12.5 and E13.5 [Ma et al., 1997; Casarosa et al., 1999; Eisenstat et al., 1999; Fode et al., 2000; Wullimann and Mueller, 2004a; Mueller and Wullimann, 2005]. A very similar pattern for Neurogenin-1-related and NeuroD expression in the forebrain was also seen in the African clawed-frog, Xenopus laevis [Wullimann et al., 2005]. Therefore, we assume that this early pattern of neurogenesis, as well as overall molecular organization, is highly conserved in all vertebrate forebrains. This is best exemplified by the generation of GABAergic cells [Katarova et al., 2000; Mueller et al., 2006]. At two days postfertilization, the majority of GABA cells are restricted to those regions that either express Dlx2a together with Zash1a, or express Zash1a alone, i.e., those regions that show the expression switched pattern. The first mentioned regions include the subpallium (S), the preoptic region (Po), the ventral thalamus $(\mathrm{VT})$ and the hypothalamus $(\mathrm{H})$, all of which show a massive presence of GABA cells. In addition, many GABA cells were found to originate in the Zashla-positive regions with the expression switched pattern, such as the pretectum (Pr) and the ventral posterior tuberculum (PTv). Thus, all of these regions that predominantly show GABA cells in correlation with Dlx2a and/or Zash1a expression at two days postfertilization could be safely considered as primary production sites of GABAergic cells. As early as three days postfertilization, however, we found some scattered GABA cells in the pallium of zebrafish (fig. 2); these cells are very likely not produced there but rather have tangentially migrated from a subpallial site of origin, as has been shown in mice and various other mammalian species. Thus, the overall distribution of GABA cells in zebrafish forebrains between two and three days postfertilization highly resembles the condition in mouse brains between E12.5 and E.13.5 [Katarova et al., 2000; Mueller et al., 2006]. We call this time frame a vertebrate phylotypic stage, or state of forebrain neurogenesis, because the striking similarities in zebrafish and mice likely reflect ontogenetic constraints in this and other regards. A similar situation - including delayed appearance and distribution - has been reported for GABAergic cells in the dorsal telencephalon (pallium) of lampreys [Meléndez-Ferro et al., 2002; Robertson et al., 2007]; furthermore, tangential migration of GABAergic 
cells from the subpallium into the pallium can be observed in other amniote species besides mammals [turtles, Métin et al., 2007; chicks, Cobos et al., 2001a] and in anamniotes other than zebrafish, i.e., amphibians [Brox et al., 2003; Moreno et al., 2008] and sharks [RodríguezMoldes, 2009].

All in all, the molecular organization of the forebrain in teleosts (zebrafish, at least), and the distribution of its GABA cells at an early postembryonic stage, highly resemble the situation in mammals (the mouse, at least) and reflect common ontogenetic constraints in the early production and regulation of neuronal phenotypes in vertebrates (secondary neurogenesis in anamniotes; primary neurogenesis in mammals). Outgroup comparisons with other fishes, such as lampreys [Villar-Cerviño et al., 2009] and sharks [Rodriguez-Moldes, 2009], as well as other anamniotes, such as frogs [Barale et al., 1996; Brox et al., 2003; Medina et al., 2005; Wullimann et al., 2005; Moreno et al., 2008] support the idea of a phylotypic stage of neurogenesis within vertebrate forebrains.

\section{Evolution and Development of Teleostean Basal Ganglia}

A recent comparative analysis of the adult forebrain in teleosts suggested that pallidal and striatal elements, which together comprise the telencephalic basal ganglia, are intermingled in the dorsal nucleus of the subpallium [Vd; Rink and Wullimann, 2001; Wullimann and Mueller, 2004a]. The finding in three-day-old zebrafish of possibly migrated GABA-positive cells from the subpallium into the pallium raised the question whether these cells might originate in a specialized area of the early basal ganglia that is homologous to the pallidal primordium, the medial ganglionic eminence (MGE) of mammals. In mice, the pallidal and striatal primordia are molecularly defined as specialized regions. Both primordia show expression of $D l x 2$ and Mash1, but only the pallidal primordium (MGE) expresses the LIM genes Lhx6 and $L h x 7$ [Grigoriou et al., 1998; Puelles et al., 2000; Zerucha et al., 2000; Yun et al., 2002; Zhao et al., 2003]. The GABAergic interneurons of the cortex in adult mice are produced in the MGE and they invade the pallium via tangential migration around E13.5. This tangential migratory stream is visible in histological preparations for in situ hybridization of GAD67 and $L h x 7$, where expressing cells are found in the pallium and the LGE [Sussel et al., 1999; Puelles et al., 2000; Anderson et al., 2001; Zhao et al., 2003, 2008].

An Evolutionary Interpretation of

Teleostean Forebrain Anatomy
In order to determine the early molecular organization of larval teleostean brains, we looked at the expression patterns of Dlx $2 a, \operatorname{Lh} x 6, \operatorname{Lh} x 7$, and GAD67 in the brains of two- to three-day-old zebrafish [Mueller et al., 2008], i.e., those stages that were shown to correspond to the brains of mice at stages E12.5-E13.5 [Mueller and Wullimann, 2005]. Again, we found that the molecular organization of the basal ganglia in zebrafish was strikingly similar to that in mammals (fig. 2). One has to take into account the partial eversion of the telencephalon in teleosts [Wullimann and Mueller, 2004a], which leads to different topographical locations of these markers in teleosts and mammals. The Dlx $2 a$ gene was found to be expressed in all subpallial regions close to the proliferative periventricular matrix, but not in radially migrated territories such as the lateral subpallium (M4; primordial lateral nucleus of the area ventralis). Thus, at two and three days postfertilization, the expression of $D l \times 2 a$ serves as a subpallial marker, just like pallial markers such as Tbr2 (eomesa), and subsequently allowing identification of the pallial-subpallial border (PSB). The $\operatorname{Lh} x 6$ gene was found to be expressed in most of the subpallium, including postmitotic and radially migrating cells and radially migrated subpallial derivatives, such as the lateral subpallium (M4), but it appeared to be expressed only in the ventral, not in the dorsal, territory of the dorsal subpallium (Sdv), and not in tangentially migrating cells. Similar to $L h x 6$, the $L h x 7$ gene is expressed in a periventricular portion of the subpallium that is also marked by $D l \times 2$, namely the ventral territory of the dorsal subpallium (Sdv), which likely demarcates the medial ganglionic eminence (MGE), plus the subpallial septal primordium in larval teleosts. The synthetic enzyme for GABA, GAD67 (glutamic acid decarboxylase) was found to be expressed in all forebrain regions previously shown to contain GABAergic cells, i.e., the subpallium, preoptic region, pretectum, ventral thalamus, hypothalamus, and posterior tuberculum [Mueller et al., 2006], but important differences were noted with respect to the telencephalon. For example, at three days postfertilization, GAD67 is expressed in most postmitotic radially migrated cells of the dorsal and ventral subpallium in a pattern similar to that of the GABA-positive cell distribution. In addition, GAD67-expressing cells are found in the migrated area of the lateral subpallium (M4), which is GABA-negative at two and three days. Other than in the septal region (Sv), most GAD67-expressing cells, close to or overlapping the proliferative subpallial matrix, were found in the ventral part of the dorsal subpallium (Sdv), with only a few such cells in the dorsal part of the dorsal subpallium

Brain Behav Evol 2009;74:30-42 
(Sdd). The GAD67-expressing cells of the ventral part of the dorsal subpallium (Sdv) form a tangentially oriented strip of cell masses. This strip comprises periventricularly located cells that overlap with the proliferative matrix in Sdv and continue into a large GAD67-positive cell mass, bypassing the only weakly GAD67-positive proliferative and postmitotic cells of the dorsal part of the dorsal subpallium (Sdd), and crossing the pallial-subpallial boundary (PSB) into the pallium. The number of GAD67expressing cells in the pallium is considerably higher than that of GABA cells at this stage. This distribution of GAD67-expressing cells in the developing zebrafish brain clearly resembles the subpallial-pallial migratory stream of GABAergic cells found in mammals, thus indicating the presence of a teleostean subpallial region homologous to mammalian MGE; both are the major source of tangentially migrating cell masses, which apparently give rise to pallial GABAergic interneurons in teleost fish (zebrafish) and mammals (mouse). As is the case for the LGE in mice [Anderson et al., 2001], a minor contribution of tangentially migrating GABA cells, destined for the cortex, might come from the Sdd in zebrafish. Notably, the subpallial-pallial tangential migratory stream of GABAergic cells into the pallium in teleosts takes a topographically medial route, whereas its counterpart in mammals takes a lateral route, thus reflecting the respective topological relationship of the subpallium and the pallium in the everted teleostean telencephalon and the evaginated mammalian telencephalon, respectively. It is another striking example of an evolutionarily conserved developmental mechanism in two vertebrate forebrains that appear very different morphologically.

\section{Identification and Morphogenesis of the Eminentia Thalami (EmT) in Teleosts}

Puelles and Rubenstein [1993] originally defined the larval eminentia thalami (EmT) as being the most posterior, alar-plate-derived portion of the secondary prosencephalon (prosomere 4), located immediately anterior to the ventral thalamus (prosomere 3 ), but they recently revised this definition to include the EmT in the most dorsal part of (prethalamic) prosomere 3 [Puelles and $\mathrm{Ru}-$ benstein, 2003]. The embryonic EmT in mammals is molecularly defined by periventricular expression domains of neurogenin $1 / 2$ and Pax6 and, more laterally, by NeuroD and Tbr1/2 domains, as well as by calretinin expression [Abbott and Jacobowitz, 1999; Bulfone et al., 1999; Puelles et al., 2000; Englund et al., 2005; Osório et al., 2009].
Thus, the molecular characteristics of the EmT clearly differ from those of the caudally adjacent ventral thalamus and those of the anteriorly adjacent preoptic region, both of which are defined by the expression of $D l \times 2$ and Mash1, thus indicating that the EmT derivatives in adults are likely glutamatergic regions or nuclei. Recently, it was suggested that the adult derivatives might comprise the bed nucleus of the stria medullaris and/or the bed nucleus of the stria terminalis [Puelles et al., 2000; Cobos et al., 2001b].

We identified a larval teleostean eminentia thalami (EmT) in zebrafish, based on a comparative analysis of expression patterns of ngn1, NeuroD, Pax6 and Tbr2 [Wullimann and Mueller, 2004b]. In particular, our twoto three-day larvae showed radially migrating streams of cells expressing NeuroD between the preoptic region and the ventral thalamus [thus matching the topological organization described in hamsters by Keyser, 1972] and extending towards the periphery of the pia surrounding the lateral forebrain bundle. A weaker, less extensive expression pattern for neurogenin1 has already been discussed as possibly representing the EmT [Mueller and Wullimann, 2003]. We then compared the NeuroD-expression patterns with corresponding histological sections from three-day-old zebrafish larvae stained with an antibody against Hu-proteins, which is a marker for differentiating neurons. Considered together, these data revealed the location and anatomical delineation of the EmT in larval teleosts [Wullimann and Mueller, 2004b]. As in the EmT in embryonic mammals, the larval EmT in teleosts is molecularly defined by the expression of $n g n 1 / 2$, and Pax6 (periventricularly), as well as NeuroD (see above) and Tb1/2 [Puelles et al., 2000; Mione et al., 2001; Wullimann and Mueller, 2004b; Englund et al., 2005; Osório et al., 2009]. Furthermore, as in mammals, the larval EmT in teleosts is sandwiched between the Dlx2(a)- and Zash1a- (Mash1-)expressing ventral thalamus, caudally, and the Dlx2(a) and Zash1a- (Mash1-)expressing preoptic region rostrally [Puelles et al., 2000; Mueller et al., 2008].

Due to new data on the distribution of GAD67-mRNA in the forebrain of adult zebrafish [Mueller and Guo, 2009] as well as data on the related gene expression patterns (Neurogenin2, NeuroD, Mash1) in embryonic mouse brains [Osório et al., 2009], we now have additional clues regarding the adult derivatives of the EmT in zebrafish as well as in mice. In contrast to our initial hypothesis that the zebrafish (teleostean) EmT gives rise to the entire entopeduncular complex [Wullimann and Mueller, 2004b], it now appears that only the GAD67-negative ventral en- 
Fig. 3. Schematic drawings of transverse sections through the forebrain of an early zebrafish (left) and mouse at a comparable stage (right) showing expression patterns of ngn1/neuroD (black) and Tbr2 (hatched). Radial migratory streams of cells originating in the eminentia thalami (EmT) are heading towards a location near the lateral forebrain bundle (zebrafish) or cerebral peduncle (mouse). The interrupted dashed gray lines in both sketches indicate the hypothetical separation of the alar hypothalamic domain from the thalamic eminence domain. [Left panel after Mione et al., 2001; Wullimann and Muller, 2004a; Mueller et al., 2008. Right panel after $\mathrm{Pu}-$ elles et al., 2000; Englund et al., 2005; Osório et al., 2009]. See list for abbreviations.

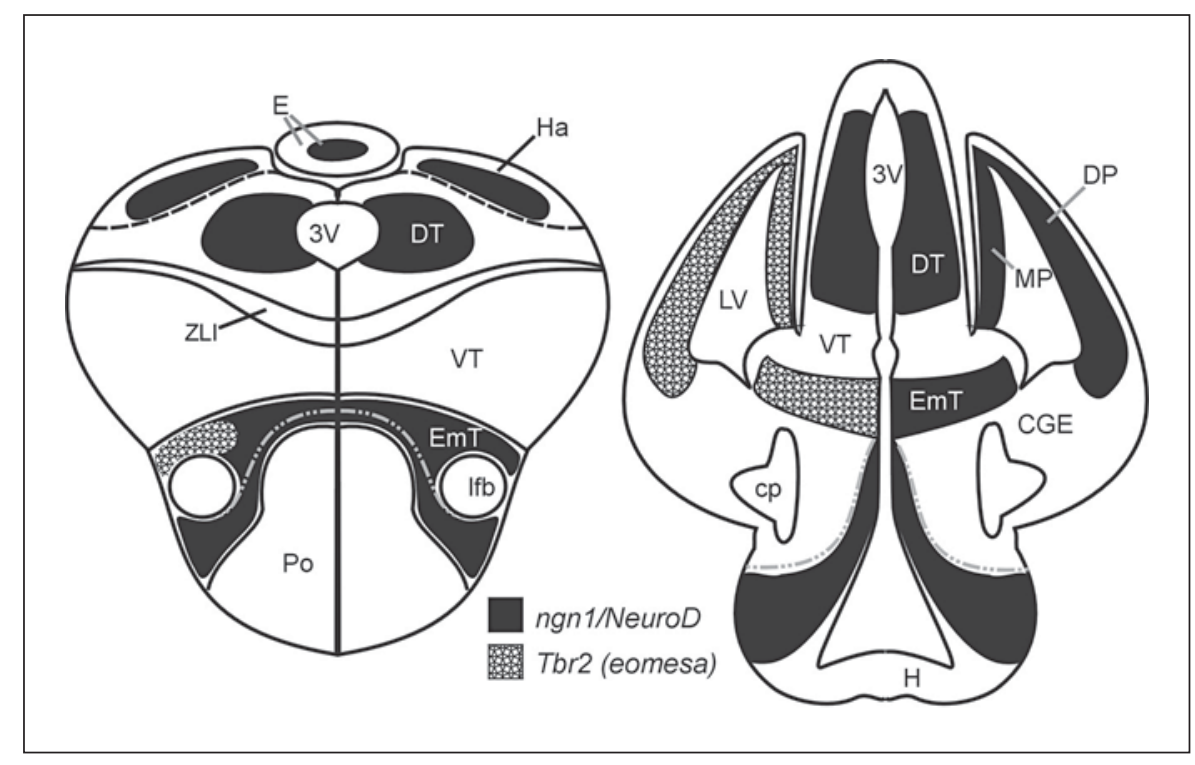

topeduncular nucleus is derived from the larval EmT, whereas the GAD67-expressing dorsal entopeduncular nucleus is not likely to be a derivative of EmT [Mueller and Guo, 2009]. The EmT-derived GAD67-negative ventral entopeduncular nucleus is probably homologous to the bed nucleus of the stria medullaris (BNSM) in mammals [Mueller and Guo, 2009]. In support of this hypothesis is the recent finding that this nucleus is calretininpositive, as is the developing EmT in mammals [Abbott and Jacobowitz, 1999] and the discovery that it projects to the habenula [Hendricks and Jesuthasan, 2007]. Also, the BNSM in mammals is characterized by the presence of many cells immunoreactive for enkephalin but very few cells immunoreactive for GAD67 [Risold and Swanson, 1995], a condition consistent with the assumption that the majority of BNSM neurons are glutamatergic. Therefore the GAD67-positive dorsal portion of the zebrafish entopeduncular complex (i.e., only the former dorsal entopeduncular nucleus) is assumed to be homologous to the entopeduncular nucleus proper in non-primate mammals and the internal segment of globus pallidus in primates [Mueller and Guo, 2009]. It is likely that this part of the entopeduncular complex also projects to the habenula, but this projection should be devoid of calretinin-expression and should express parvalbumin instead [Rajakumar et al., 1994; Yáñez and Anadon, 1996].

As a novel point of interest, our recent mouse data [Osório et al., 2009] revealed that a strip of neurogenin2expressing cells extends peripherally from the ventricular location where the EmT domain emerges (fig. 3). This strip clearly leads into the territory of the hypothalamus (which is otherwise Mash1-positive) and reaches the pial periphery anterior to the cerebral peduncle (fig. 3). We interpret this ventrolateral ngn2 stream as representing part of the alar hypothalamus in mice [Puelles and $\mathrm{Ru}-$ benstein, 2003]. Interestingly, and consistent with this hypothesis, a part of the alar-plate-derived hypothalamus also remains GAD-free [Katarova et al., 2000]. In comparison, the described strips of NeuroD- and ngn1expressing cells in developing larvae of three-day-old zebrafish originate at the ventricle where the EmT is located, and they also extend posteriorly and anteriorly to the lateral forebrain bundle, thus topologically almost mirroring the situation in mice (fig. 3). The ventral stream in zebrafish might therefore include glutamatergic cells from the alar hypothalamus that have an unknown topographical destiny, in addition to cells that contribute to the bed nucleus of the stria medullaris, which in adult zebrafish is located both anterior and posterior to the lateral forebrain bundle [Mueller and Guo, 2009].

\section{Evolution and Development of the Teleostean Pallium}

The organization of the pallium in adult teleosts, as well as its developmental and and evolutionary interpretation, has for years been under debate [Nieuwenhuys, 1963, 2009; Northcutt and Braford, 1980; Northcutt and Davis, 1983; Nieuwenhuys and Meek, 1990; Costagli et 

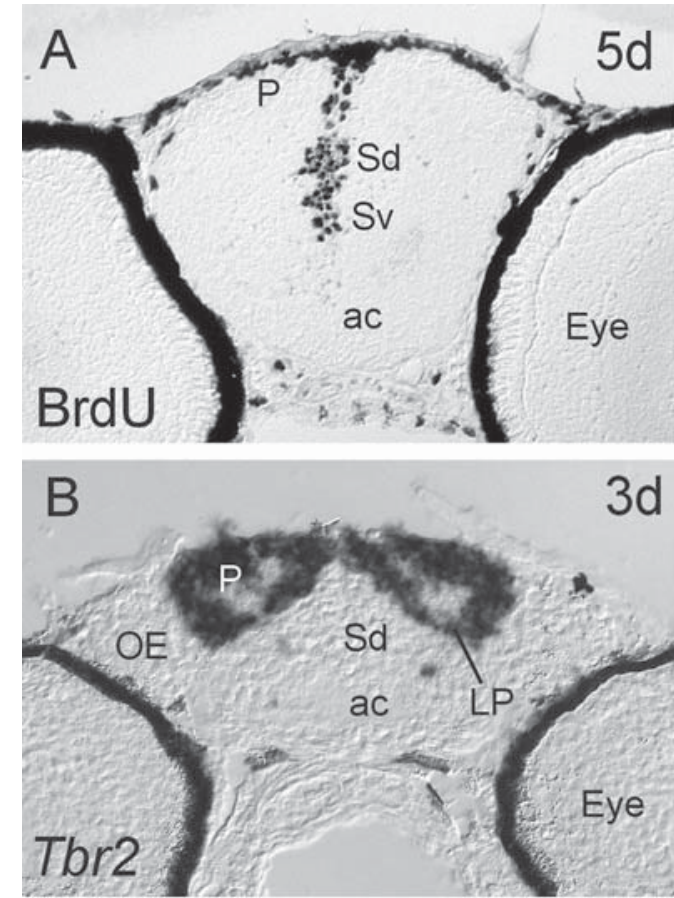

proliferative cells

Tbr2 (eomesa)
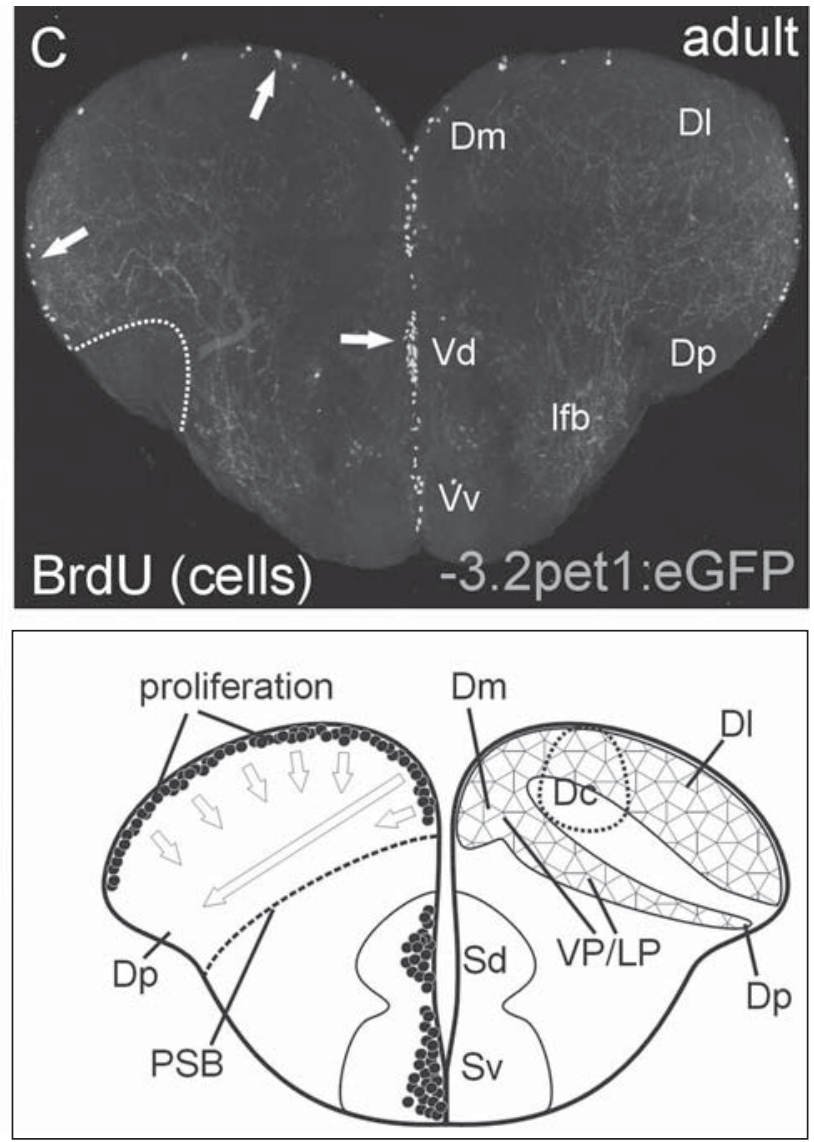

Fig. 4. Zebrafish pallial organization. A Photomicrograph shows telencephalic proliferation at 5 days postfertilization, with BrdU distribution demonstrating pallial eversion. B The expression domains of Tbr2 (eomesa) in the 3-day-old zebrafish telencephalon form a dorsal and a ventral tier. The distinct ventral tier of Tbr2expressing cells can be interpreted as radially migrating cells of the ventral and lateral pallium, which form in the outermost periphery the future posterior zone of the dorsal telencephalic area (Dp; lateral pallium homologue). Schematic drawing at right bottom summarizes these data. C Photomicrograph of a section through the telencephalon of an adult transgenic zebrafish $\operatorname{Tg}(-3.2$ pet1:eGFP) showing serotonin-positive fibers (stained by green fluorescent protein, GFP, appearing light gray). Also shown is proliferation with BrdU-positive cells (white). Note that, in contrast to everted pallial zones, like the medial zone (Dm) and the lateral zone (Dl) of the dorsal telencephalon, as well as the subpallial ventral nucleus $(\mathrm{Vv})$ and dorsal nucleus (Vd) of the ventral telencephalon, the surface of the posterior zone of the dorsal telencephalic area (Dp) in the adult brain does not appear to contain BrdU-positive proliferating cells, supporting the view that this surface is pial, not ventricular (see text). See list for abbreviations. (C is from Lillesaar et al. [2009]; original photograph courtesy of Christina Lillesaar and Laure Bally-Cuif). al., 2002; Northcutt, 2006, 2008]. The eversion of pallial masses in teleosts, in contrast to their evagination in most other vertebrates, results in a different topography in the telencephalon. We have proposed an explicit hypothesis of partial eversion in the telencephalon of teleosts that combines morphogenetic and molecular developmental data [Wullimann and Mueller, 2004a]. In brief, evagination results in two telencephalic hemispheres that enclose a central (bilateral) ventricle and show the basic gnathostome arrangement of subpallial and pallial mass- es (seen in fig. 2, right panel), In partial eversion, the subpallial masses lie medial, with the pallium having rolled out laterally (seen in fig. 2, left panel), taking with it the fusion point (roof plate) of the medial pallium and stretching the roof plate into an epithelial covering of the thinned out ventricle. In developing mouse embryos, gene expression patterns of the pallial genes Emx1, Tbrl, Pax6 and $D b x 1$ (the last of which is selective for the ventral pallium and its derivatives) indicated that the pallium in mammals is comprised of four divisions [Puelles et al., 1999; 
Medina et al., 2004; Englund et al., 2005]: (1) the medial pallium, which gives rise to the hippocampus; (2) the dorsal pallium, which gives rise to the six-layered isocortex; (3) the ventral pallium, which gives rise to parts of the pallial amygdaloid nuclei (basolateral and lateral amygdala), plus parts of the endopiriform and claustral areas; and (4) the lateral pallium, which gives rise to the piriform cortex and other parts of the claustroamygdaloid complex. Similar gene expression data for teleost fish have long been missing, but molecular, hodological, and behavioral data from adult teleosts (mainly goldfish and zebrafish) meanwhile suggest the existence of four teleost pallial divisions, homologous to the medial, dorsal, ventral and lateral pallium [Mueller and Wullimann, 2005]. Teleostean pallial organization can best be explained with a partial eversion model (see above) [Wullimann and Mueller, 2004a], because such a model assumes that the olfactory pallium (posterior zone of dorsal telencephalic area; Dp) is homologous to the lateral pallium (piriform cortex) in mammals and is a migrated derivative of a ventricular region assumed to lie within the midline portion of the medial zone of area dorsalis telencephali $(\mathrm{Dm})$ in teleosts. In support of this assumed origin is that the posterior (olfactory) pallium (Dp) does not show a superficial zone of proliferation, in contrast to other pallial divisions (Dm, Dl), which - due to eversion - have their proliferative ventricular matrix zone at the surface of the telencephalon. The absence of a superficial zone of proliferation was demonstrated very clearly in adult zebrafish (fig. 4C) after a short BrdU incubation [Lillesaar et al., 2009] and suggests that - in contrast to the medial and lateral zones of area dorsalis telencephali - the posterior zone has no ventricular proliferative zone at the surface and therefore does not appear to be everted. The attachment of the tela choroidea, however, remains disputable. We hypothesized in our partial eversion model, that this attachment lies at the peripheral boundary of the posterior and lateral zones of area dorsalis. According to Northcutt and Davis [1983], this is the case in the gar Lepisosteus and in a teleost, Salmo, but not in the teleost Lepomis, where the attachment lies at the ventrolateral border of the posterior zone of area dorsalis. We have unfortunately not been able to show the attachment of the telencephalic tela, directly, in our own preparations. Regardless of this issue, another line of evidence supports the assumed mediolateral migration of pallial cell masses into the posterior zone of area dorsalis (Dp). As mentioned above, the proliferative zone of $\mathrm{Dp}$ is hypothesized to be in the midline of the medial zone of area dorsalis in adult teleosts, presumably dorsal to the ventral pallium

An Evolutionary Interpretation of

Teleostean Forebrain Anatomy
[VP = precursor of much of the pallial amygdala, which is assumed to represent much or all of Dm in adults; Northcutt, 2006]. The partial eversion model predicts a radial migratory stream towards the pial surface of the posterior zone of area dorsalis (Dp). The expression pattern of Tbr2 [Mueller et al., 2008] is consistent with this assumption and indicates a radial migratory stream from the midline of the pallium towards the pial surface, where the posterior zone of area dorsalis $(\mathrm{Dp})$ comes to lie in adults. This represents the first evidence for the presence of a lateral pallial migratory stream and supports our pallial eversion model [Wullimann and Mueller, 2004a], as well as the suggestion that the posterior zone of area dorsalis (Dp) is homologous to the lateral pallium of other vertebrates (including the piriform cortex of mammals). There is substantial connectional evidence [Northcutt, 2006] and lesion-dependent behavioral evidence [Salas et al., 2003; Portavella et al., 2004] that Dm is homologous to the pallial amygdala (ventral pallium derivative) and that $\mathrm{Dl}$ is homologous to the hippocampus (medial pallium derivative).

\section{Conclusions}

In the context of our interest in the structure and evolution of vertebrate brains, we use the zebrafish as a genetic model in which we can hopefully decipher the development, organization, and evolution of the forebrain in a teleost fish. Comparison of our data from teleosts with those from a mammal (the mouse) provides further insights into vertebrate evolution and, ultimately, the molecular basis of human neurological disorders. In our review, the similarities in forebrain development in zebrafish and mice are often striking. Do these similarities reflect on ancestral developmental constraints leading to similar solutions? Did we show that there are more homologous areas and nuclei in mammalian and teleostean forebrains than previously thought? And are these homologous areas/nuclei functionally equivalent to their mammalian counterparts? Clearly, many questions remain, mostly because the condition in many other vertebrate groups has not been examined, and some of the homologies suggested in the forebrains of zebrafish and mice could turn out to represent examples of convergence, with similarities that are independently derived. Information from Xenopus, however [Medina et al., 2005; Wullimann et al., 2005], and, importantly, newly emerging data from cartilaginous fishes [Rodríguez-Moldes, 2009] indicate that many of the developmental events we 
describe herein represent a generalized condition in vertebrate brains. At the same time, the noted absence of markers for a structure homologous to the medial ganglion eminence/primordial pallidum in lampreys [Osório et al., 2005] indicates that some of the patterns described herein originated with gnathostomes and might not be present in agnathans. We believe that the search for developmental similarities and early gene activity is more promising than the comparison of adult brains, because it takes into account the neural origin of structures in a Bauplan of histogenetic units, which is relatively stable compared to the range of variation seen in the adult morphology among various species. Furthermore, our approach goes beyond morphogenetic issues (such as eversion), as we directly target the genetic control of de- velopmentally relevant events in neurogenesis. The molecular markers that we use allow us to differentiate the spatial origin of major neuronal phenotypes and their early anatomical patterns of distribution and migration. This ontogenetic approach is therefore qualitatively different from previously applied comparisons based on adult topography alone, which can lead to false conclusions when examining brains of animals as phylogenetically divergent as mammals and teleost fishes.

\section{Acknowledgement}

We would like to thank Mary Sue Northcutt for substantial editorial and stylistic improvement of the manuscript.

\section{References}

Abbott LC, Jacobowitz DM (1999) Developmental expression of calretinin-immunoreactivity in the thalamic eminence of the fetal mouse. Int J Dev Neurosci 17:331-345.

Allende ML, Weinberg ES (1994) The expression pattern of two zebrafish achaete-scute homo$\log (a s h)$ genes is altered in the embryonic brain of the cyclops mutant. Dev Biol 166: 509-530.

Anderson SA, Marín O, Horn C, Jennings K, Rubenstein JLR (2001) Distinct cortical migrations from the medial and lateral ganglionic eminences. Development 128:353-363.

Anderson SA, Qiu M, Bulfone A, Eisenstat DD, Meneses J, Pedersen R, Rubenstein JLR (1997). Mutations of the homeobox genes Dlx- 1 and $D l x-2$ disrupt the striatal subventricular zone and differentiation of the late born striatal neurons. Neuron 19:27-37.

Appel B, Eisen JS (1998) Regulation of neuronal specification in the zebrafish spinal cord by Delta function. Development 125:371-380.

Barale E, Fasolo A, Girardi E, Artero C, Franzoni MF (1996) Immunohistochemical investigation of gamma-aminobutyric acid ontogeny and transient expression in the central nervous system of Xenopus laevis tadpoles. J Comp Neurol 368:285-294.

Bertrand N, Castro DS, Guillemot F (2002) Proneural genes and the specification of neural cell types. Nat Rev Neurosci 3:517-530.

Blader P, Fischer N, Gradwohl G, Guillemot F, Strähle U (1997) The activity of neurogenin1 is controlled by local cues in the zebrafish. Development 124:4557-4569.

Brox A, Puelles L, Ferreiro B, Medina L (2003) Expression of the genes GAD67 and Distalless-4 in the forebrain of Xenopus laevis confirms a common pattern in tetrapods. J Comp Neurol 461:370-393.
Bulfone A, Martinez S, Marigo V, Campanella M, Basile A, Quaderi N, Gattuso C, Rubenstein JLR, Ballabio A (1999) Expression pattern of the Tbr2 (Eomesodermin) gene during mouse and chick brain development. Mech Dev 84:133-138.

Casarosa S, Fode C, Guillemot F (1999) Mash1 regulates neurogenesis in the ventral telencephalon. Development 126:525-534.

Cobos I, Puelles L, Martínez S (2001a) The avian telencephalic subpallium originates inhibitory neurons that invade tangentially the pallium (dorsal ventricular ridge and cortical areas). Dev Biol 239:30-45.

Cobos I, Shimamura K, Rubenstein JLR, Martínez S, Puelles L (2001b) Fate map of the avian anterior forebrain at the four-somite stage, based on the analysis of quail-chick chimeras. Dev Biol 239:46-67.

Costagli A, Kapsimali M, Wilson SW, Mione M (2002) Conserved and divergent patterns of reelin expression in the zebrafish central nervous system. J Comp Neurol 450:73-93.

Dornseifer P, Takke C, Campos-Ortega JA (1997) Overexpression of a zebrafish homologue of the Drosophila neurogenic gene Delta perturbs differentiation of primary neurons and somite development.. Mech Dev 63:157-171.

Eisenstat DD, Liu JK, Mione M, Zhong W, Yu G, Anderson SA, Ghattas I, Puelles P, Rubenstein JL (1999) $D L X-1, D L X-2$, and $D L X-5$ expression define distinct stages of basal forebrain differentiation. J Comp Neurol 414: 217-237.

Englund C, Fink A, Lau C, Pham D, Daza RAM, Bulfone A, Kowalcyk T, Hevner RF (2005). Pax6, Tbr2, and Tbr1 are expressed sequentially by radial glia, intermediate progenitor cells, and postmitotic neurons in developing neocortex. J Neurosci 25:247-251.
Flames N, Pla R, Gelman DM, Rubenstein JL, Puelles L, Marín O (2007) Delineation of multiple subpallial progenitor domains by the combinatorial expression of transcriptional codes. J Neurosci 27:9682-9695.

Fode C, Ma Q, Casarosa S, Ang SL, Anderson DJ, Guillemot F (2000) A role for neural determination genes in specifying the dorsoventral identity of telencephalic neurons. Genes Dev 14:67-80.

Gould SJ (1977) Ontogeny and phylogeny. Cambridge, MA: Harvard University Press.

Grigoriou M, Tucker AS, Sharpe PT, Pachnis V (1998) Expression and regulation of $\operatorname{Lhx} 6$ and $L h x 7$, a novel subfamily of LIM homeodomain encoding genes, suggests a role in mammalian head development. Development 125:2063-2074.

Hack MA, Saghatelyan A, de Chevigny A, Pfeifer A, Ashery-Padan R, Lledo PM, Götz M (2005) Neuronal fate determinants of adult olfactory bulb neurogenesis. Nat Neurosci 8: 865-872.

Hendricks M, Jesuthasan S (2007) Asymmetric innervation of the habenula in zebrafish. J Comp Neurol 502:611-619.

Horton S, Meredith A, Richardson JA, Johnson JE (1999) Correct coordination of neuronal differentiation events in ventral forebrain requires the bHLH factor Mash1. Mol Cell Neurosci 14:355-369.

Katarova Z, Sekerková G, Prodan S, Mugnaini E, Szabó G (2000) Domain-restricted expression of two glutamic acid decarboxylase genes in midgestation mouse embryos. J Comp Neurol 424:607-627.

Keyser A (1972) The development of the diencephalon of the Chinese hamster. Acta Anat 83 (Suppl 59):1-181. 
Korzh V, Slepsova I, Liao J, He J, Gong Z (1998) Expression of zebrafish bHLH genes ngn1 and nrd defines distinct stages of neural differentiation. Dev Dyn 213:92-104.

Lledo PM, Alonso M, Grubb MS (2006) Adult neurogenesis and functional plasticity in neuronal circuits. Nat Rev Neurosci 7:179193.

Lillesaar C, Stigloher C, Tannhäuser B, Wullimann MF, Bally-Cuif L (2009) Axonal projections of raphe serotonergic neurons in the developing and adult zebrafish, Danio rerio, visualized by transgenic demonstration of raphe-specific pet1 expression. J Comp Neurol 512:158-182.

Ma Q, Sommer L, Cseriesi P, Anderson DJ (1997) Mash1 and neurogenin1 expression patterns define complementary domains of neuroepithelium in the developing CNS and are correlated with regions expressing Notch ligands. J Neurosci 17:3644-3652.

Marín O, Rubenstein JLR (2001) A long, remarkable journey: tangential migration in the telencephalon. Nat Rev Neurosci 2:780-790.

Marín O, Anderson SA, Rubenstein JLR (2000) Origin and molecular specification of striatal interneurons. J Neurosci 20:6063-6076.

Medina L, Legaz I González G, de Castro F, Rubenstein JLR, Puelles L (2004) Expression of Dbx1, Neurogenin 2, Semaphorin 5A, Cadherin 8, and Emxl distinguish ventral and lateral pallial histogenetic divisions in the developing mouse claustroamygdaloid complex. J Comp Neurol 474:504-523.

Medina L, Brox A, Legaz I, García-López M, Puelles L (2005) Expression patterns of developmental regulatory genes show comparable divisions in the telencephalon of Xenopus and mouse: insights into the evolution of the forebrain. Brain Res Bull 66:297-302.

Meléndez-Ferro M, Pérez-Costas E, Villar-Cheda B, Abalo XM, Rodríguez-Muñoz R, Rodicio MC, Anadón R (2002) Ontogeny of-aminobutyric acid-immunoreactive neuronal populations in the forebrain and midbrain of the sea lamprey. J Comp Neurol 446:360376.

Métin C, Alvarez C, Moudoux D, Vitalis T, Pieau C, Molnár Z (2007) Conserved pattern of tangential neuronal migration during forebrain development. Development 134:1527.

Mione M, Shanmkugalingam S, Griffin K (2001) Overlapping expression of zebrafish T-brain1 and eomesodermin during forebrain development. Mech Dev 100:93-97.

Moreno N, González A, Rétaux S (2008) Evidences for tangential migrations in Xenopus telencephalon: developmental patterns and cell tracking experiments. Dev Neurobiol 68 504-520.

Mueller T, Guo S (2009) The distribution of GAD67-mRNA in the adult zebrafish (teleost) forebrain reveals a prosomeric pattern and suggests previously unidentified homologies to tetrapods. J Comp Neurol: in press.
Mueller T, Wullimann MF (2002) BrdU-, neuroD $(n r d)$ - and $\mathrm{Hu}$-studies reveal unusual nonproliferative neurogenesis in the postembryonic zebrafish forebrain. Mech Dev 117:123135.

Mueller T, Wullimann MF (2003) Anatomy of neurognesis in the early zebrafish brain. Brain Res Dev Brain Res 140:137-155.

Mueller T, Wullimann MF (2005) Atlas of early zebrafish brain development: a tool for molecular neurogenetics. Amsterdam: Elsevier Publishing Company.

Mueller T, Vernier P, Wullimann MF (2006) A phylotypic stage in vertebrate brain development: GABA cell patterns in zebrafish compared with mouse. J Comp Neurol 494:620634.

Mueller T, Wullimann MF, Guo S (2008) Early teleostean basal ganglia development visualized by zebrafish Dlx2a, Lhx6, Lhx7, Tbr2 (eomesa), and GAD67 gene expression. J Comp Neurol 507:1245-1257.

Nery S, Fishell G, Corgin JG (2002) The cauda ganglionic eminence is a source of distinct cortical and subcortical cell populations. Nat Neurosci 5:1279-1287.

Nieuwenhuys R (1963) The comparative anatomy of the actinopterygian forebrain. J Hirnforsch 6:171-200.

Nieuwenhuys R (2009) The forebrain of actinopterygians revisited. Brain Behav Evol 73:229252.

Nieuwenhuys R, Meek J (1990) The telencephalon of actinopterygian fishes. In: Cerebral cortex (Jones EG, Peters A, eds), pp 31-73. New York: Plenum Press.

Northcutt RG (1984) Evolution of the vertebrate central nervous system: patterns and processes. Am Zool 24:701-716.

Northcutt RG (2006) Connections of the lateral and medial divisions of the goldfish telencephalic pallium. J Comp Neurol 494:903943.

Northcutt RG (2008) Forebrain evolution in bony fishes. Brain Res Bull 75:191-205.

Northcutt RG, Braford MR Jr (1980) New observations on the organization and evolution of the telencephalon of actinopterygian fishes. In: Comparative neurology of the telencephalon (Ebbesson SOE, ed), pp 41-98. New York: Plenum Press.

Northcutt RG, Davis RE (1983) Telencephalic organization in ray-finned fishes. In: Fish neurobiology, Vol. 2. Higher brain areas and functions. Ann Arbor (Davis RE, Northcutt RG, eds), pp 203-236. Ann Arbor, MI: University of Michigan Press.

Osório J, Mazan S, Rétaux S (2005) Organisation of the lamprey (Lampetra fluviatilis) embryonic brain: insights from LIM-homeodomain, Pax and hedgehog genes. Dev Biol 288: $100-112$.

Osório J, Mueller T, Rétaux S, Vernier P, Wullimann MF (2009) Phylotypic expression of the bHLH genes neurogenin2, neuroD and Mash1 in the mouse embryonic forebrain. J Comp Neurol: in press.
Panganiban G, Rubenstein JLR (2002). Developmental functions of the Distal-less/Dlx homeobox genes. Development 129:43714386.

Portavella M, Torres B, Salas C (2004). Avoidance response in goldfish: emotional and temporal involvement of medial and lateral telencephalic pallium. J Neurosci 24:23352342.

Puelles L, Medina L (2002) Field homology as a way to reconcile genetic and developmental variability with adult homology. Brain Res Bull 57:243-255.

Puelles L, Rubenstein JLR (1993) Expression patterns of homeobox and other putative regulatory genes in the embyonic mouse forebrain suggests a neuromeric organization. Trends Neurosci 16:472-479.

Puelles L, Rubenstein JLR (2003) Forebrain gene expression domains and the evolving prosomeric model. Trends Neurosci 26:469-476.

Puelles L, Kuwana E, Puelles E, Rubenstein JLR (1999) Comparison of the mammalian and avian telencephalon from the perspective of gene expression data. Eur J Morphol 37:139150

Puelles L, Kuwana E, Puelles E, Bulfone A, Shimamura K, Keleher J, Smiga S, Rubenstein JLR (2000) Pallial and subpallial derivatives in the embryonic chick and mouse telencephalon, traced by the expression of the genes Dlx-2, Emx-1, Nkx-2.1,Pax-6, and Tbr1. J Comp Neurol 424:409-438.

Rajakumar N, Elisevich K, Flumerfelt BA (1994) Parvalbumin-containing GABAergic neurons in the basal ganglia output system of the rat. J Comp Neurol 350:324-336.

Rink E, Wullimann MF (2001) The teleostean (zebrafish) dopaminergic system ascending to the subpallium (striatum) is located in the basal diencephalon (posterior tuberculum) Brain Res 889:316-330.

Risold PY, Swanson LW (1995) Cajal's nucleus of the stria medullaris: characterization by in situ hybridization and immunohistochemistry for enkephalin. J Chem Neuroanat 9: 235-240.

Robertson B, Auclair F, Menard A, Grillner S, Dubuc R (2007) GABA distribution in lamprey is phylogenetically conserved. J Comp Neurol 503:47-63.

Rodríguez-Moldes I (2009) A developmental approach to forebrain organization in elasmobranchs: New perspectives on the regionalization of the telencephalon. Brain Behav Evol 74:20-29.

Ross SE, Greenberg ME, Stiles CD (2003) Basic helix-loop-helix factors in cortical development. Neuron 39:13-25.

Salas C, Broglio C, Rodríguez F (2003) Evolution of forebrain and spatial cognition in vertebrates: conservation across diversity. Brain Behav Evol 62:72-82.

Schuurmans C, Guillemot F (2002). Molecular mechanisms underlying cell fate specification in the developing telencephalon. Curr Opin Neurobiol 12:26-34. 
Sussel L, Marín O., Kimura S, Rubenstein JLR (1999) Loss of $N k x 2.1$ homeobox gene function results in ventral to dorsal molecular respecification within the basal telencephalon: evidence for a transformation of the pallidum into the striatum. Development 126: 3359-3370.

Villar-Cerviňo B, Barreiro-Iglesias A, Anadón R, Rodicio MC (2009) Development of glycine immunoreactivity in the brain of the sea lamprey: comparison with $\gamma$-aminobutyric acid immunoreactivity. J Comp Neurol 512: 747-767.

von Baer KE (1828) Über die Entwickelungsgeschichte der Thiere. Beobachtung und Reflexion. Königsberg: Bornträger.

Wullimann MF, Mueller T (2002) Expression of Zash-1a in the postembryonic zebrafish brain allows comparison to mouse Mash1 domains. Brain Res Gene Expr Patt 1:187192.

Wullimann MF, Mueller T (2004a) Teleostean and mammalian forebrains contrasted: evidence from genes to behavior. J Comp Neurol 475:143-162.
Wullimann MF, Mueller T (2004b) Identification and morphogenesis of the eminentia thalami in the zebrafish. J Comp Neurol 471: 37-48.

Wullimann MF, Puelles L (1999) Postembryonic neural proliferation in the zebrafish forebrain and its relationship to prosomeric domains. Anat Embryol 199:329-348.

Wullimann MF, Rink E (2002) The teleostean forebrain: a comparative and developmental view based on early proliferation, Pax6 activity and catecholaminergic organization. Brain Res Bull 57:363-370.

Wullimann MF, Rink E, Vernier P, Schlosser G (2005) Secondary neurogenesis in the brain of the African clawed frog, Xenopus laevis, as revealed by PCNA, Delta-1, Neurogenin-related-1 and NeuroD expression. J Comp Neurol 489:387-402.

Yáñez J, Anadon R (1996) Afferent and efferent connections of the habenula in the rainbow trout (Oncorhinus mykiss): an indocarbocyanine dye (DiI) study. J Comp Neurol 372: 529-543.
Yun K, Fischman S, Johnson J, De Angelis MH, Weinmaster G, Rubenstein JLR (2002) Modulation of the notch signaling by Mash1 and $D l x 1 / 2$ regulates sequential specification and differentiation of progenitor cell types in the subcortical telencephalon. Development 129:5029-5040.

Zerucha T, Stühmer T, Hatch G, Park BK, Long QM, Yu GY, Gambarotta A, Schultz JR, Rubenstein JLR, Ekker M (2000) A highly conserved enhancer in the $D l \times 5 / D l x 6$ intergenic region is the site of crossregulatory interactions between $D l x$ genes in the embryonic forebrain. J Neurosci 20:709-721.

Zhao Y, Marín O., Hermesz E, Powell A, Flames N, Palkovits M, Rubenstein JLR, Westphal H (2003) The LIM-homeobox gene $\operatorname{Lh} x 8$ is required for the development of many cholinergic neurons in the mouse forebrain. Proc Natl Acad Sci USA 100:9005-9010.

Zhao Y, Flandin P, Long JE, Cuesta MD, Westphal H, Rubenstein JL (2008) Distinct molecular pathways for development of telencephalic interneuron subtypes revealed through analysis of Lhx6 mutants. J Comp Neurol 510:79-99. 